von Nebenwirkungen ab. Die Patienten wurden im Schnitt für 16 Monate weiter beobachtet. Sieben Patienten mussten aus verschiedenen Gründen die Weiterbehandlung mit Pramipexol einstellen. Fünf von ihnen erlitten innerhalb von zwei Wochen einen Rückfall in die Depression, nur zwei blieben symptomfrei. Die häufigsten Nebenwirkungen waren Übelkeit, Schlafstörungen und Schläfrigkeit. Die Nebenwirkungen ließen sich bei einem Teil der Patienten gut durch Dosisreduktion beherrschen. Patienten mit bipolarer und unipolarer Depression unterschieden sich nicht hinsichtlich der Ansprech- und Remissionsraten unter Pramipexol.

\section{Kommentar}

Die vorliegende Fallsammlung unterstreicht die seit langem diskutierte Bedeutung dopaminerger Therapieverfahren bei therapieresistenter Depression. Das Format der

Tab. 1: Ergebnisse von 42 Patienten mit therapieresistenter Depression, die mit Pramipexol behandelt wurden, differenziert nach Erkrankungstyp

\begin{tabular}{|l|l|l|l|l|}
\hline Gruppe & Remission & Response & Non-Response & $\begin{array}{l}\text { Behandlung nicht } \\
\text { toleriert }\end{array}$ \\
\hline Bipolare Störung $(n=18)$ & 9 & 5 & 1 & 3 \\
\hline $\begin{array}{l}\text { Unipolare Depression } \\
(n=24)\end{array}$ & 11 & 7 & 1 & 5 \\
\hline \begin{tabular}{l} 
Gesamt $(n=42)$ \\
\hline
\end{tabular} & 20 & 12 & 2 & 8 \\
\hline
\end{tabular}

Fallsammlung ermöglicht hier die Übertragung in die eigene Praxis, da die Autoren Tipps und Hinweise für die klinische Anwendung aus ihrer eigenen Erfahrung heraus geben können. Wahrscheinlich gilt für Substanzen wie Pramipexol ähnlich wie bereits für Ketamin, dass damit klinische Erfahrungen gesammelt werden müssen. Wichtig ist, dass keine dieser Substanzen in der Indikation zugelassen ist und es sich daher immer um einen ärztlichen Heilversuch mit allen seinen besonderen Anforderungen an Aufklärung und Einwilligung handelt.

Prof. Dr. med. Helge Frieling

Fawcett $J$ et al. Clinical Experience with highdosage pramipexole in patients with treatmentresistant depressive episodes in unipolar and bipolar depression. Am J Psychiatry 2016; 173: $107-11$

\title{
Gibt es ein bipolares Prodrom?
}

\begin{abstract}
Die Früherkennung von Erkrankungen aus dem schizophrenen Formenkreis möglichst bereits während eines Prodromalstadiums wird immer wichtiger. Aber gibt es so etwas wie ein spezifisches Prodromalsyndrom für bipolare Störungen? Eine aktuelle Studie aus Pittsburgh und Toronto gibt Antworten.
\end{abstract}

$\mathrm{U}$ m mögliche typische Dimensionen eines bipolaren Prodroms zu entdecken, untersuchten die Wissenschaftler 359 Kinder und Jugendliche im Alter von acht bis 16 Jahren, von denen mindestens ein Elternteil an einer bipolaren Störung litt. Diese Hochrisikopopulation wurde mit 220 Kindern und Jugendlichen aus denselben Gemeinden verglichen. Bereits zum Baseline-Zeitpunkt litten $8,4 \%$ der Hochrisikoprobanden an einer Störung aus dem bipolaren Spektrum. Am Ende des Beobachtungszeitraums von acht Jahren litten 14,7\% der Kinder, für die vollständige Daten vorhanden waren (44/299), an einer Störung aus dem bipolaren Störung; davon hatten 15 eine ausgewachsene bipolare Ioder II-Störung. Um mögliche, dem Auftreten einer Störung vorhergehende Symptome zu erfassen, wurden zum Baseline-Zeitpunkt und bei allen Folge- untersuchungen verschiedenste Daten erhoben, die mittels Faktoranalyse zu einzelnen Faktoren komprimiert wurden. Mögliche Prodromalsyndrome wurden anhand der Ausprägung der verschiedenen Faktoren zur Baseline und zur letzten Untersuchung vor der Transition zu einer ausgereiften Störung ausgewertet. Es zeigte sich, dass die Kinder in der Hochrisikopopulation höhere Baseline-Werte für die Faktoren Angst/ Depression, Unaufmerksamkeit/Disinhibierung, Externalisierung, subsyndromale Manie und Affektlabilität aufwiesen. Die beste Vorhersagekraft für das spätere Auftreten einer bipolaren Spektrumerkrankung hatten hohe BaselineWerte für Angst/Depression und Affektlabilität. Letztere sagten auch besonders gut bei der vorausgehenden Untersuchung die nahende Transition voraus, ebenso wie subsyndromale manische
Symptome. Jugendliche ohne einen dieser Faktoren hatten lediglich ein $2 \%$ iges Risiko, im Verlauf eine bipolare Spektrumserkrankung zu erleiden. Diejenigen mit allen drei Faktoren hatten eine $49 \%$ ige Wahrscheinlichkeit dafür.

\section{Kommentar}

Jugendliche, die auf dimensionalen Skalen (wie dem BDI o.ä.) hohe Werte für Angst und Depression und Affektlabilität erzielen oder bereits unter subsyndromalen manischen Symptomen leiden, haben ein besonders hohes Risiko eine bipolare Störung zu entwickeln, wenn ein Elternteil bereits darunter leidet. Obwohl diese Angaben nicht besonders spezifisch wirken, können sie sehr hilfreich bei der Risikoeinschätzung und zur klinischen Verlaufsbeurteilung bei Risikopatienten sein. Inwieweit sie sich auch bei Jugendlichen ohne genetische Belastung bewähren, müssen weitere Studien untersuchen.

Prof. Dr. med. Helge Frieling

Hafeman DM et al. Toward the Definition of a Bipolar Prodrome: Dimensional Predictors of Bipolar Spectrum Disorders in At-Risk Youths. Am J Psychiatry 2016; 173: 695-704 